\title{
100 Jahre Resektion der kompletten Trachea
}

\author{
100 Years Resection of the Complete Trachea
}

Vor genau 100 Jahren wurden in Deutschland erstmals 2 erfolgreiche Trachea-Querresektionen vorgenommen. Die Operationsmethode wurde von Themistokles Gluck (1853-1942) (@ Abb. 1) inauguriert und von ihm und seinem Schüler und engsten Mitarbeiter Johannes Soerensen am 15. Mai und 15. September 1913 bei 2 Patienten wegen stenosierender Karzinome der distalen Trachea erstmals angewandt. Die Erstbeschreibung erfolgte durch Soerensen im Archiv f. Laryngologie 1915 [1] (○ Abb. 2). In der gängigen Literatur zur Tracheachirurgie [2-5] findet die hier zusammengefasste Arbeit keine Beachtung. Umso wichtiger ist es, sie an den Beginn einer thoraxchirurgischen Serie im Zentralblatt für Chirurgie zu stellen. Es soll damit ins Bewusstsein gebracht werden, dass schon vor 100 Jahren ohne die heutigen technischen Hilfsmittel mit logischem chirurgischen Denken und Handeln, konsequenter Verfolgung eines Konzepts und profunder Kenntnis der topografischen Anatomie und Pathophysiologie zum ersten Mal chirurgische Wege für Patienten in ausweglosen Situationen gefunden und gegangen wurden. Damit sollen keine Heldentaten beschrieben oder Helden gefeiert werden, sondern ein Denkanstoß gegeben werden zur Frage, ob medizinischer Fortschritt untrennbar mit Hightech verbunden sein muss. Soerensens Artikel zeigt, dass es nicht allein neue Technik ist, die uns voranbringt, sondern denkende und handelnde Menschen, die die vorhandene Technik beherrschen.

\section{Operationstechnik}

Johannes Soerensen beschreibt auf 17 Seiten die Kasuistiken zweier Patienten, 44 und 32 Jahre alt, die beide bei einer malignen Stenose der Luftröhre unter lebensbedrohlichen Atemnotzuständen litten und in das „Kaiser und Kaiserin Friedrich-Krankenhaus“ in Berlin eingeliefert wurden. In beiden Fällen wurde die
Atemnot - wie auch heute noch häufig zunächst als Asthma fehldiagnostiziert und behandelt.

Nach tracheoskopischer Sicherung eines endotrachealen Tumors, dessen Ausdehnung nach distal nicht festgestellt werden konnte, hat man mit der damals wie heute gängigen Methode einer Tracheotomie (in Lokalanästhesie) und Einlage einer Trachealkanüle die Stenose zunächst überbrückt: „In dem ersten der beiden Fälle mussten wir eine lange biegsame Hummerschwanzkanüle mit ziemlich grosser Gewalt durch die stenosierte Stelle hindurchschieben." Dabei wurde in einem Fall ein endoluminaler Tumorblock akzidentell „abgehobelt“ und damit der Atemweg zunächst wieder freigelegt. Histologisch wurde beides Mal ein Karzinom festgestellt.

Die Patienten befanden sich also nach der Tracheotomie in einer Palliativsituation. Soerensen und Gluck hatten jedoch bei der Tracheotomie sowohl mit einem Rinnenspekulum, einmal aber auch digital einen tumorfreien Rand zwischen distalem Tumorende und Bifurkation festgestellt. Damit schienen theoretisch die Voraussetzungen für eine onkologisch radikale Operation mit querer Resektion der gesamten Trachea gegeben. Der Entschluss dazu fiel unter den Gesichtspunkten, dass es sich jeweils um einen nicht metastasierten, fokalen Tumor handelte, der den Ösophagus nicht infiltriert hatte (Ösophagoskopie) und dem altersbezogen geringen allgemeinen Operationsrisiko der beiden Patienten.

Drei wesentliche Erkenntnisse aus früheren Eingriffen am Kehlkopf und der oberen Luftröhre waren für Gluck und Soerensen die Grundlage zur Querresektion der Trachea:

- Die in Einzelfällen sehr gute Mobilisierbarkeit der Trachea und der Bifurkation.

- Die eigenen Erfolge bei der Deckung kollarer Tracheadefekte mit Hautlappen.

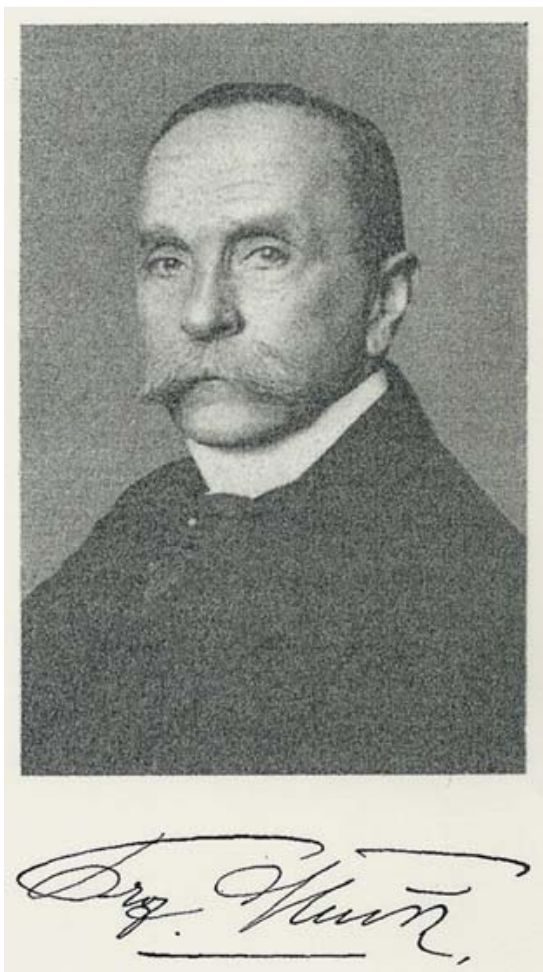

Abb. 1 Themistokles Gluck 1853-1942.

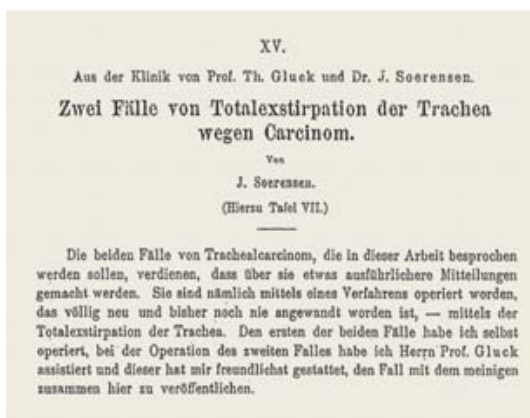

Abb. 2 Erstbeschreibung der kompletten Trachearesektion 1915.

- Die Idee, schrittweise vorangehen zu können und palliative Alternativen zur Querresektion bereit zu haben.

Soerensen hat den ersten Eingriff trotz der damals vehement vertretenen Lehrmeinung gewagt, dass Querresektionen nur am oberen Tracheadrittel möglich seien:

„... Der untere... Teil des Organs galt bisher den Chirurgen so sehr als ein Nolimetangere, dass die Autoren nicht einmal die Möglichkeit äußerer Eingriffe in dieser Gegend gelten lassen. ... Worin die angenommenen unüberwindlichen Schwierigkeiten bei Operationen am unteren Tracheateil eigentlich bestehen, darüber werden nirgends nähere Angaben ge- 


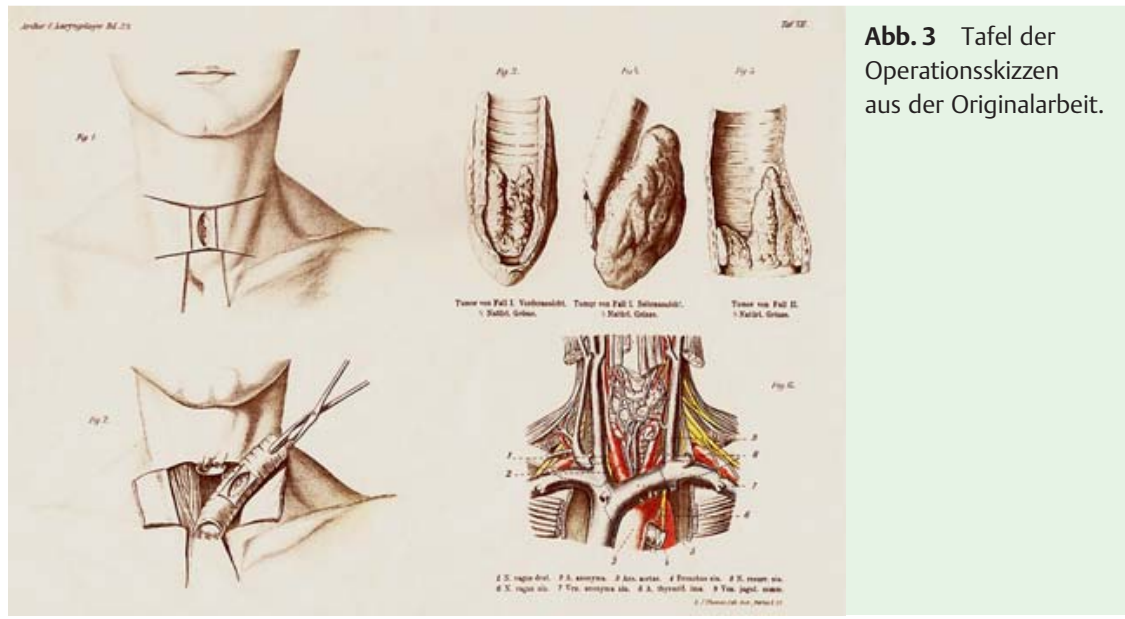

macht. Ich darf jedoch wohl annehmen, dass man der Ansicht war, dass es

1. sehr schwierig sei, sich ohne Nebenverletzungen das eigentliche Operationsgebiet freizulegen,

2. dass man keinen sicheren Weg sah, während der Operation das Einfliessen von Blut in die tieferen Luftwege zu verhindern,

3. dass es unmöglich sei, nach Exstirpation des unteren Teiles der Trachea die Wunde so zu versorgen, dass die freie Zufuhr der Atmungsluft gewährleistet und das Herabfliessen von Wundsekret mit seinen verderblichen Folgen gehindert würde.“

Offensichtlich hatten Gluck und Soerensen aber Lösungen für diese Schwierigkeiten bei vorangegangenen Operationen und im Tierversuch erarbeitet.

Etwa 2 Wochen nach der Tracheotomie folgte dann der Versuch der kompletten Tracheaquerresektion. Nach Umschneiden des Tracheostomas und größtenteils stumpfer peritrachealer Präparation mit Auslösen der extramuralen Tumoranteile en bloc ließ sich die Bifurkation schrittweise nach kranial mobilisieren. Die größte Herausforderung sahen die beiden Pioniere darin, die Retraktion der Bifurkation intraoperativ, aber auch im Falle einer denkbaren postoperativen Komplikation zu verhindern, was unmittelbar zur Aspiration und Asphyxie geführt hätte. „Es empfiehlt sich nicht, die Trachea in einem Zuge quer abzuschneiden, weil man sich damit jeden Haltes an der Bifurkation beraubt. Es würde nicht ganz leicht sein, die letztere so lange mittels Zangen oder anderer Instrumente im Niveau der Wunde zu halten, bis man die Lappen ringsherum angarniert hätte.“ Statt Klemmen oder Zangen beließ man also einfach die tumortragende Trachea nach größtenteils stumpfer Auslösung derselben aus dem Mediastinum als Zuginstrument an der Bifurkation.

Es war Soerensen zu diesem Zeitpunkt der Operation nicht klar, „... ob man nun in der Lage sein würde, diesen Zug ... zu verstärken, ohne durch den Zug an Bronchien, Gefässen und Herzbeutel die Respiration und Blutzirkulation in grober Weise zu schädigen...“. „... es blieb zunächst zweifelhaft, ob nicht ein stärkeres, forziertes Anziehen der Trachea bedenkliche Störungen im Gefolge haben würde. Diese Befürchtungen sind ... erfreulicherweise nicht eingetreten."

Nachdem sichergestellt wurde, dass der vorpräparierte ventrale Hautlappen weit genug über das Jugulum nach retrosternal reichte, um ihn mit der Vorderwand der Bifurkation zu anastomosieren, wurde dort die Trachea auf $1 / 3$ ihrer Zirkumferenz inzidiert und die tracheokutane Anastomose mit dem über das Manubrium gezogenen Hautlappen „angarniert“. Damit war der Luftweg gesichert. Es folgte die Durchtrennung der einen Tracheaseitenwand am Tracheobronchialwinkel, die mit dem seitlichen Hautlappen des Halses anastomosiert wurde. Erst jetzt trennte man auch die letzte Verbindung zwischen Trachea und Bifurkation und konnte das Präparat mit dem Tumor bergen, der beim 1. Patienten als „gänseeigroß“ beschrieben wurde ( $\triangle$ Abb. 3, oben rechts). Nach Vervollständigung der tracheokutanen Anastomose waren nur noch die 3 Hautlappen durch Längsnähte zu verbinden. Damit war ein Luftröhrenersatz geschaffen, der in einem weiten Tracheostoma unmittelbar oberhalb des Jugulums mündete. „Wenn die Wunde geheilt ist, ist der Endeffekt folgender: man sieht vorn am Hals eine längsgestellte elliptische Oeffnung, durch die man

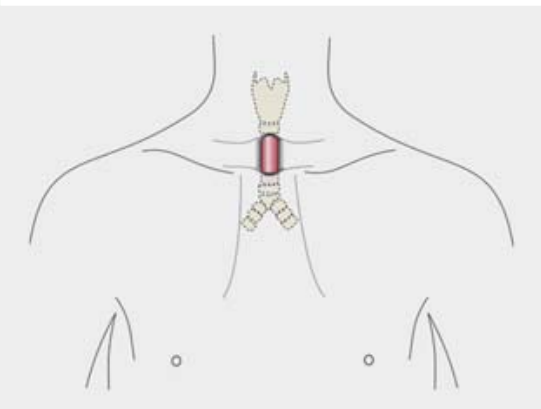

Abb. 4 Skizze postoperativ mit Gummikanüle.

nach unten in das neue Trachealrohr bis zur Abgangsstelle der Bronchien hinabsieht....".

Bei gesundem Kehlkopf galt es nun, dessen Funktion wieder herzustellen. Dazu wurden die Ränder des Ringknorpels mit der Haut des Halses vernäht:, ... während man nach aufwärts von unten her in den Kehlkopf hineinblickt.“ Die phonetische Funktion des Kehlkopfs war nun allein durch Abdecken des neu entstandenen „plastischen Tracheostomas“ wieder gegeben. Allerdings in einem Fall mit Heiserkeit wegen einer Läsion des rechtsseitigen N. laryngeus recurrens inferior. Für eine bessere Wiederherstellung der Kontinuität besann man sich auf „alloplastisches Ersatzmaterial“" in Form eines Gummiröhrchens, das in den proximalen und distalen Schenkel des Tracheostomas eingeführt (und mutmaßllich mit einem seidenen Halstuch unter dem Hemdkragen bedeckt) wurde ( Abb. 4). „Es belästigt den Träger in keiner Weise und braucht zum Zweck der Reinigung alle 24 Stunden gewechselt zu werden.“

Als postoperative Komplikation wird einzig eine Wundheilungsstörung an einer kutanobifurkalen Naht beschrieben. Die Katastrophe der Retraktion der Bifurkation blieb jedoch aus, da die Nähte der beiden anderen Hautlappen primär geheilt waren und die Spannung aufnehmen konnten.

Die histologische Bearbeitung der beiden Präparate hat in einem Fall eine ektope Struma maligna und im anderen Fall ein primäres Tracheakarzinom ergeben. Onkologisch ist in beiden Fällen eine R0-Resektion gelungen, sodass während eines 2-jährigen Bobachtungszeitraums bei keinem Patienten ein Rezidiv auftrat. Eine noch längere Nachbeobachtung war sicherlich durch die Wirren des Ersten Weltkriegs nicht gegeben. 


\section{Diskussion}

Die Beschreibung der beiden Operationen und der nicht nur zu erahnenden Begleitumstände in der Sprache des 19. Jahrhunderts ist es wert, im Original gelesen $\mathrm{zu}$ werden. Man darf mutmaßen, dass ein so enormer Fortschritt der Chirurgie heute medial ungleich größer aufgemacht daherkäme als in einer bescheidenen wissenschaftlichen Publikation.

Erst in den 60er-Jahren des 20. Jahrhunderts wird wieder über Trachea- und $\mathrm{Bi}$ furkationsresektionen mit End-zu-EndAnastomosen berichtet. Experimentelle Arbeiten entstanden, bei denen der langstreckige Luftwegdefekt nach kompletten Trachearesektionen durch alloplastisches Material in situ überbrückt werden sollte. Regelmäßig kam es dabei jedoch zu Insuffizienzen an den Anastomosen, weshalb dieser Weg nicht weiter verfolgt wurde [6].

Auch heute gehören Trachearesektionen nicht nur wegen der seltenen Indikation zu Eingriffen, die einer besonderen Expertise des Operateurs und der Klinik bedürfen:

- Chirurgen, die Tracheaeingriffe vornehmen, müssen damals wie heute neben den reinen resezierenden Verfahren mit End-zu-End-Anastomose auch die interventionellen endoluminalen Behandlungstechniken (endobronchiale Abtragung, Laser, Stent) kennen, besser selbst beherrschen und deren Vor- und Nachteile gegeneinander abwägen können [7].

- Das Team muss im Zusammenspiel zwischen Anästhesie und Thoraxchirurgie (ggf. auch Kardiotechnik) die intraoperative Oxygenierung sicherstellen können und im Falle intraoperativer Komplikationen eine Alternative „im Hinterkopf“ haben [8].

- In der Phase der intensiv- und postoperativen Therapie müssen die spezielle postoperative Problematik wie Glottisödem, vulnerable Anastomose und Sekretstau bekannt sein und individuelle Lösungskonzepte interdisziplinär geplant und umgesetzt werden können.

- Es müssen interventionelle Konzepte für Anastomoseninsuffizienzen und -stenosen existieren.

Die Biografie von Themistokles Gluck [9] lässt keine Zweifel aufkommen, dass er und Johannes Soerensen prädestiniert waren, auf diesem Gebiet eine Vorreiterrolle zu übernehmen. Es war kein Zufall, dass sie beiden Patienten mit der neuen Operationstechnik in einer nahezu aus- weglosen Situation eine langfristige Perspektive geben konnten. Das Interesse und die Arbeiten Glucks auf dem Gebiet der Transplantation und dem Organersatz aller Organe deuten darauf hin, dass dieses Chirurgenteam schon vor über 100 Jahren das Potenzial und die Ideen hatte, die erst heute erreichten Fortschritte des autologen und homologen Tracheaersatzes voranzubringen. Das wurde erst 100 Jahre später mit Ersatzgewebe aus Aortenwand kombiniert mit formstabiliserenden passageren Stents oder mit Transplantaten von lyophilisierter Leichentrachea erzielt $[10,11]$.

Ob die für erfolgreiches Tissueengineering [12] notwendige Kontrolle der Zellproliferation und die Kombination unterschiedlicher Gewebetypen (Knorpel, Bindegewebe und zilientragende Mukosa) für einen sicheren Tracheaersatz möglich werden, wird erst die Zukunft zeigen. Es ist nicht ausgeschlossen, dass Glucks Ansatz mit Haut als autologem Ersatzgewebe in Zukunft wieder aufgegriffen wird und wir zu den Prinzipien der inzwischen 100 Jahre alten Lösung zurückkommen werden.

Die Medizingeschichte von damals zeigt, dass - vielleicht mehr als heute - synergistische Begleitumstände notwendig waren, um in der Forschung erfolgreich zu sein. Das Studium der biografischen Literatur legt nahe, dass Gluck nicht so sehr von diesen Begleitumständen profitieren konnte wie andere Chirurgen seiner Zeit [9]. Mutmaßlich war dieser Umstand zusammen mit der Seltenheit der Indikation der Trachearesektion die Ursache dafür, dass Weiterentwicklungen auf diesem Gebiet in den folgenden Jahrzehnten ein Schattendasein in der chirurgischen Forschung fristeten.

Heute dagegen ist oft mangelnde Kommunikation zwischen den Fachdisziplinen bedingt durch die Topografie der Trachea zwischen Hals (HNO), Lunge (Thoraxchirurgie und Pneumologie), Ösophagus (Viszeralchirurgie), Herz und thorakalen Gefäßen (Herzchirurgie) ein Grund dafür dass selbst bei der adäquaten Versorgung von Erkrankungen der Trachea Nachholbedarf besteht. Zu Soerensens und Glucks Zeiten war die damals noch nicht existierende Spezialisierung für die Tracheachirurgie zweifellos ein Vorteil, da Chirurgen zu jener Zeit keine regionalen Grenzen einhalten mussten. Das darf aber nicht als Kritik an der heutigen Spezialisierung verstanden werden, sondern vielmehr als Appell zu mehr interdisziplinärem Dialog, wenn es um die Behand- lung von Tracheaerkrankungen und deren Weiterentwicklung geht.

Gluck und Soerensen haben bereits vor einhundert Jahren gezeigt, dass Haut als autologes Gewebe für eine sichere Verbindung zwischen den Grenzflächen an Bifurkation und Krikoid sorgen kann. Die Kombination mit alloplastischem Material (Gummi) stellte den Lufttransport via obere Luftwege durch Hals und Mediastinum in die Lunge sicher. Die Erkenntnis, dass es transplantierbares, der Tracheawand vergleichbares, körpereigenes Gewebe nicht gibt, zwang sie schließlich zu der beschriebenen Kombinationslösung. Wenn man Glucks bekanntgemachte $\mathrm{Ar}$ beiten aber liest, dann ist es keine kühne Hypothese anzunehmen, dass auch die Idee des gezüchteten Gewebeersatzes schon damals in seinem Geist existierte. Soerensens „vergessene“ Publikation verdient eine häufigere Zitation allein aus Ehrfurcht und Anerkennung der chirurgischen Leistung der beiden Chirurgen auf diesem Gebiet und dem Gebiet der Thoraxchirurgie überhaupt. Gluck war wie viele andere Forscher der zeitgenössischen Wissenschaft oft weit voraus, weshalb u.a. seine Arbeiten über anatomische Lungenresektionen an Tieren von den damaligen chirurgischen Meinungsbildnern vehement kritisiert wurden. Chirurgisch muss man Gluck als universell bezeichnen, denn sein Interesse und seine Forschungsarbeiten reichten von der Chirurgie des Larynx, der Trachea und des Ösophagus über die Neurochirurgie, die Allgemeinchirurgie bis hin zur Transplantation und dem Gelenk- und Sehnenersatz. J. Zange [9] beschreibt seine Rolle in der damaligen chirurgischen Welt wie folgt: „Für den Fernerstehenden erscheint es unbegreiflich, wie ein an kühnen und originellen Ideen so reicher Geist wie Gluck, der den größten chirurgischen Problemen seiner Zeit mit solchem Erfolge nachging, solange ohne gebührende Anerkennung blieb, dass darüber sein weiterer beruflicher Lebensweg der eines Außenseiters wurde, statt dass er in führende Stellung gelangte. ... er musste mit anderen Großen der Wissenschaft das Schicksal langer Verkennung teilen, weil es zu neue und ungewohnte Dinge waren, die er brachte....".

So liegt es vielleicht auch in seinem Genie begründet, dass er vergaß, Johannes Soerensen darauf hinzuweisen, zumindest einen Absatz der Publikation der Narkose und der Sauerstoffzufuhr zu widmen und uns nicht nur mit dem Satz: „Dann wurde der Kranke bei hochgelagertem Oberkör- 
per und rückwärtsgebeugtem Kopf tief narkotisiert..." darüber im Ungewissen zu lassen, wie die Patienten während dieser sicherlich mehrstündigen Operation geschlafen und geatmet haben.

Wir haben daher geplant, im nächsten historischen Artikel zur Thoraxchirurgie eine ausführliche Analyse der damals möglichen Narkoseführung und (Be-)Atmung darzustellen; denn man kann nicht einerseits den interdisziplinären Dialog fordern und andererseits zu diesem wichtigen Thema nicht den Anästhesisten zu Wort kommen lassen, um diese offen gebliebene Frage zu beantworten.

\section{Interessenkonflikt: Nein}

A. Linder ${ }^{1}$, A. Busemann², P. Menges ${ }^{2}$

1 Thoraxchirurgie, Klinikum Bremen Ost, Bremen, Deutschland

2 Klinik für Viszeral-, Thorax- und Gefäßchirurgie, Universitätsmedizin Greifswald, Greifswald, Deutschland

\section{Literatur}

1 Soerensen J. Zwei Fälle von Totalexstirpation der Trachea wegen Karzinom. Archiv Laryngol Rhinol 1915; 29: 188-204

2 Maasen W, Greschuchna D, Vogt-Moykopf I et al. Tracheal resection - state of the art. Thorac Cardiovasc Surg 1985; 33: 2-7

3 Grillo HC. Development of tracheal surgery: a historical review. Part 1: techniques of tracheal surgery. Ann Thorac Surg 2003; 75: 610-619

4 Grillo HC. Development of tracheal surgery: a historical review. Part 2: treatment of tracheal diseases. Ann Thorac Surg 2003; 75 : 1039-1047

5 Pearson FG, Cooper JD, Nelems JM et al. Primary tracheal anastomosis after resection of the cricoid cartilage with preservation of recurrent laryngeal nerve. J Thorac Cardiovasc Surg 1975; 70: 806-816

6 Toomes H, Mickisch G, Vogt-Moykopf I. Experiences with prosthetic reconstruction of the trachea and bifurcation. Thorax 1985; 40: 32-37

7 Herth F J, Freitag $L$. Interventionelle Therapie beim endobronchialen Tumorbefall Laser, Stent, Brachytherapie und anderes. Der Onkologe 2005; 11: 759-768

8 Männle C, Layer M, Vogt-Moykopf I et al. Hochfrequenz-Jet-Beatmung während Trachearesektion bei Kindern und Säuglingen. AINS 1997; 32: 21-26
9 Zange J. Th. Gluck zum Gedächtnis. Archiv f. Ohren-, Nasen- und Kehlkopfheilkunde 1942; 151: 101-106

10 Wurtz A, Porte H, Conti $M$ et al. Surgical technique and results of tracheal and carinal replacement with aortic allografts for salivatory gland type carcinoma. J Thorac Cardiovasc Surg 2010; 140: 387-393

11 Probst EJ, Prager JD, Meinzen-Derr J et al. Pediatric tracheal reconstruction using cadaveric homograft. Arch Otolaryngol Head Neck Surg 2011; 137: 583-590

12 Bader A, Macchiarini P. Moving towards in situ tracheal regenaration: the bionic tissue engineered transplantation approach. J Cell Mol Med 2010; 14: 1877-1889

\section{Bibliografie}

Dol http://dx.doi.org/10.1055/s-1328268

Zentralbl Chir 2013; 138: 107-110

(c) Georg Thieme Verlag KG Stuttgart · New York . ISSN 0044-409X

\section{Korrespondenzadresse}

Prof. Albert Linder, MD

Thoraxchirurgie

Klinikum Bremen Ost

Züricher Str. 40

28325 Bremen

Deutschland

dr.a.linder@gmail.com 